\title{
Conditional Recurrence-Free Survival for Patients with Gallbladder Cancer: A Personalized Resource for Patients and Providers
}

\author{
Aslam Ejaz, MD, MPH, and Timothy M. Pawlik, MD, PhD, MPH (1D \\ Department of Surgery, The Ohio State University Wexner Medical Center, James Cancer Center, Columbus, OH
}

Gallbladder cancer is an aggressive biliary tract cancer in which surgical resection provides the only hope for longterm survival. However, even among eligible patients who undergo curative-intent surgical resection, 5-year overall survival (OS) is generally dismal, ranging from 5 to $26 \%$. The wide variation in OS is largely due to the presence of certain adverse pathologic features at the time of surgery, such as advanced $\mathrm{T}$ - or $\mathrm{N}$-disease. Estimation of long-term outcome using these factors alone can be problematic as these data are "static" and do not take into account individual dynamic prognostic factors over time. To this point, previous research from our own group, as well as others, has demonstrated that traditional estimates for OS rely too heavily on static risk factors determined at the time of surgery and are therefore disproportionately influenced by patients who die shortly following surgery. ${ }^{1-3}$ Given this, conditional survival (CS), which accounts for the time a patient has already survived following surgery/diagnosis, has been proposed as a more accurate estimate of long-term survival. ${ }^{1,4,5}$ In effect, the longer an individual has survived from treatment, the greater the chance of additional survival. In the current study, ${ }^{6}$ Vega et al. reported on the conditional recurrence-free survival (RFS) of patients following extended resection of gallbladder cancer.

Utilizing an international multi-institutional database, the authors analyzed conditional RFS estimates based on 484 patients who underwent curative-intent resection for $\geq$

(C) Society of Surgical Oncology 2021

First Received: 7 January 2021

Accepted: 12 January 2021;

Published Online: 2 February 2021

A. Ejaz, MD, MPH

e-mail: Aslam.ejaz@osumc.edu
T1b gallbladder cancer. Median actual RFS among the entire cohort was 32 months, and not surprisingly, patients with higher disease stage had shorter actual RFS. RFS converged, however, after patients were recurrence free for 36 months. Furthermore, after 24 months of survival, T3T4 disease was the only factor independently associated with increased risk of recurrence. These data are interesting in light of work previously published by our own group. Specifically, similar to Vega et al., ${ }^{6}$ we had previously demonstrated that the odds of survival changed over time following resection of gallbladder cancer. For example, while actuarial survival decreased over time, conditional survival compared with 5-year actuarial survival increased after surgery. ${ }^{7}$ Unlike the current study on RFS, we also noted that positive margin status, higher tumor grade, residual disease at repeat resection, and lymph node metastasis had an impact on conditional OS. ${ }^{1}$

Data from the study by Vega et al. confirm previous reports on the utility of conditional survival to estimate prognosis among patients with gallbladder cancer. ${ }^{1,2,8}$ In particular, conditional survival estimates may help individualize surveillance patterns for patients who undergo resection of gallbladder cancer. Current NCCN guidelines recommend surveillance with cross-sectional imaging every 3-6 months for 2 years, followed by 6-12 months up to 5 years for all patients with resected gallbladder cancer. The current study demonstrated, however, that the risk of recurrence varied among patients, as individuals with T3T4 disease had a high risk of recurrence even after 24 months. In turn, conditional survival data may help to tailor surveillance programs along a more patient-specific timeline relative to an individual's risk of recurrence. Conditional survival on risk of disease recurrence may also potentially inform use of adjuvant therapy. While the use of adjuvant systemic therapy for patients with gallbladder 
cancer remains debated, these data provide a rationale for systemic therapy among patients at highest risk of recurrence based on conditional RFS risk. Furthermore, conditional survival data allow for evidence-based discussions with patients regarding their "true" individualized risk of recurrence. This is an area of need as previous studies have noted that patients often have a more optimistic perception about cure following cancer surgery. ${ }^{9}$ In fact, previously published mixture cure models have demonstrated that only approximately $15 \%$ of gallbladder cancer patients may achieve a statistical cure after surgery. ${ }^{10}$ Data from the current study provide an important framework and analytic approach to obtain individualized data-based estimates on long-term outcomes.

The study by Vega et al. ${ }^{6}$ has several limitations that should be noted. Data on CA19-9 levels were not complete and thus could not be included in the analyses. Given that CA19-9 has been demonstrated to be an important prognostic factor for conditional OS following gallbladder cancer resection, the lack of this tumor marker was problematic. ${ }^{7}$ Furthermore, only approximately one-half of the cohort had at least six lymph nodes examined, thus suggesting possible incomplete staging among these patients. ${ }^{11}$ The overall low median number of lymph nodes evaluated may have contributed to the lack of statistical significance regarding lymph node metastasis and conditional RFS.

In conclusion, we commend the authors for their work on this needed and important patient-centric study. Future studies should seek to incorporate molecular markers and genetic mutational analyses in addition to pathologic factors to improve individual patient-specific therapeutic options and prognostic stratification of patients with gallbladder cancer.

DISCLOSURE The authors declare no conflicts of interest.

\section{REFERENCES}

1. Kim Y, Ejaz A, Spolverato G, et al. Conditional survival after surgical resection of gastric cancer: a multi-institutional analysis of the us gastric cancer collaborative. Ann Surg Oncol. 2015;22(2):557-64.

2. Cucchetti A, Piscaglia F, Cescon M, et al. Conditional survival after hepatic resection for hepatocellular carcinoma in cirrhotic patients. Clin Cancer Res. 2012;18(16):4397-405.

3. Spolverato G, Kim Y, Ejaz A, et al. Conditional probability of long-term survival after liver resection for intrahepatic cholangiocarcinoma: a multi-institutional analysis of 535 patients. JAMA. 2015;150(6):538-45.

4. Wang SJ, Emery R, Fuller CD, Kim JS, Sittig DF, Thomas CR. Conditional survival in gastric cancer: a SEER database analysis. Gastric Cancer. 2007;10(3):153-8.

5. Ito Y, Nakayama T, Miyashiro I, Ioka A, Tsukuma H. Conditional survival for longer-term survivors from 2000-2004 using population-based cancer registry data in Osaka, Japan.BMC Cancer. 2013;13:304.

6. Vega EA, Newhook TE, Kawaguchi Y, et al. Conditional recurrence-free survival after oncologic extended resection for gallbladder cancer: an international multicenter analysis. Ann Surg Oncol. 2021. https://doi.org/10.1245/s10434-021-09626-3.

7. Buettner S, Margonis GA, Kim Y, et al. Changing odds of survival over time among patients undergoing surgical resection of gallbladder carcinoma. Ann Surg Oncol. 2016;23(13):4401-9.

8. Mayo SC, Nathan H, Cameron JL, et al. Conditional survival in patients with pancreatic ductal adenocarcinoma resected with curative intent. Cancer. 2012;118(10):2674-81.

9. Kim Y, Winner M, Page A, et al. Patient perceptions regarding the likelihood of cure after surgical resection of lung and colorectal cancer. Cancer. 2015;121(20):3564-73.

10. Spolverato G, Bagante F, Ethun CG, et al. Defining the chance of statistical cure among patients with extrahepatic biliary tract cancer. World J Surg. 2017;41(1):224-31.

11. Tsilimigras DI, Hyer JM, Paredes AZ, et al. The optimal number of lymph nodes to evaluate among patients undergoing surgery for gallbladder cancer: correlating the number of nodes removed with survival in 6531 patients. $J$ Surg Oncol. 2019;119(8):1099-107.

Publisher's Note Springer Nature remains neutral with regard to jurisdictional claims in published maps and institutional affiliations. 